\title{
DesCRIPTION DE DEUX NOUVELles eSPÈCES DE PHLÉbOTOMES DU SOUS-geNRE HELCOCYRTOMYIA, DU PIÉMONT ANDIN BOLIVIEN (Diptera, Psychodidae)
}

\author{
TORRES-ESPEJO J.M.* CACERES G.A.** \& LE PONT F.***
}

Summary : DESCRIPTION OF TWO NEW SPECIES OF THE HELCOCYRTOMYIA SUBGENUS FROM THE BOLIVIAN ANDEAN FOOTHILIS (Diptera, Psychodidae)

Two new species of phlebotomine sand flies from subandean region of Bolivia (alt. : 400-2000 m) are described. Both belong to the Helcocyrtomyia subgenus which includes the vectors of "uta". Lutzomyia guderiani n. sp., of the sanguinaria group, is an anthropophilic species of primary forest and coffee plantations in the Yungas. L. rispaili n. sp., described from males, is a species closely related to the pervensis group; it was caught in subandean primary forest of Alto Beni, below $1000 \mathrm{~m}$.

KEY WORDS : sand fly. Phlebotominae. taxonomy. Lutzomyia guderiani $n$. sp. Lutzomyia rispaili n. sp. Bolivia.

\section{INTRODUCTION}

'identification de Leishmania peruviana, agent de l'uta au Pérou (Arana et al., 1990), a relancé l'étude du sous-genre Helcocyrtomyia dans lequel se situent les vecteurs de ce parasite. Sept nouvelles espèces ont été décrites (Caceres et Galati, 1988; Blancas, Caceres et Galati, 1989; Galati et Caceres, 1990; Galati et Caceres, 1992; Galati et Caceres, 1994). Le sous-genre a été divisé en trois séries : sanguinaria, osornoi et peruensis et une clef d'identification des espèces a été proposée par Galati et Caceres (1994). Nous donnons ci-après la description de deux nouvelles espèces récoltées dans le piémont andin de Bolivie, l'une dans les Yungas, l'autre dans les cordillères subandines de l'Alto Beni.

Les descriptions ont été faites sur des spécimens montés dans l'Euparal. Les mensurations, en millimètres, sont celle de l'holotype et de l'allotype, tandis qu'entre parenthèses sont donnés les extrêmes des variations chez les paratypes.

\footnotetext{
* IBBA, Entomologie, casilla 717, La Paz, Bolivie.

** Institut Nacional de Salud n¹400, APO 451, Lima 100, Pérou

*** ORSTOM, Département Santé, 213 Rue de La Fayette, 75480 Paris Cedex 10.
}

\section{Résumé}

Les auteurs décrivent deux nouvelles espèces de phlébotomes de région subandine de Bolivie. (alt. : 400-2000 m). Elles appartien nent au sous-genre Helcocyrtomyia, dont font partie les vecteurs d'"uta" au Pérou. Lutzomyia guderiani n. sp., qui appartient au groupe sanguinaria, est une espèce anthropophile de forêt primaire et de caféières dans les Yungas. L. rispaili n. sp., décrite du seul sexe mâle, est apparentée au groupe peruensis; elle n'a été récoltée qu'en forêt primaire subandine de l'Alto Beni, au-dessous de $1000 \mathrm{~m}$.

MOTS CLES : phlébotome. Phlebotominae, taxonomie. Lutzomyia guderiani n. sp. Lutzomyia rispaili n. sp. Bolivie.

\section{LUTZOMYIA (HELCOCYRTOMYIA) GUDERIANI N. SP.}

(Planche I)

Description de l'holotype male (paratypes, $\mathrm{n}=13$ )

Grand phlébotome mesurant environ 3,3 mm (3,33,9) du bout du labre à l'extrémité du coxite, de coloration brun clair, plus pâle dans la partie haute des pleures.

Tête :

Longueur $0,44(0,427-0,480)$ incluant le court clypeus; largeur (max.) 0,435 (0,404-0,490). Rapport $\mathrm{L} / 1=1,02$. Distance interoculaire 0,068 (0,065-0,081), étroite, égale au diamètre de 3 facettes. Labre épipharynx, longueur $0,331(0,313-0,360)$ à partir du bord du clypeus. Flagellomères antennaires : AIII $=0,429(0,387-$ $0,465), \mathrm{AIV}+\mathrm{AV}=0,175+0,173$. Rapport $\mathrm{AIII} / \mathrm{E}=$ 1,29 (1,19-1,37) (fig. 3). Ascoïdes simples, dont l'extrémité dépasse la moitié du segment AIV (fig. 4). Palpes : longueur totale 0,709 (0,692-0,834), longueurs respectives des palpomères : 1 - $0,040(0,036-$ $0,052)$ 2- $0,161(0,138-0,177) 3-0,189(0,18-0,228) 4-$ $0,059(0,059-0,098) 5-0,260(0,234-0,295)$ (fig.3). Formule palpaire : 1.4.2.3.5. Cibarium présentant une plage de 20 à 30 denticules distribués sur trois rangées irrégulières; arc chitineux diffus dans sa partie médiane, aire pigmentée marquée, étroite et échan- 

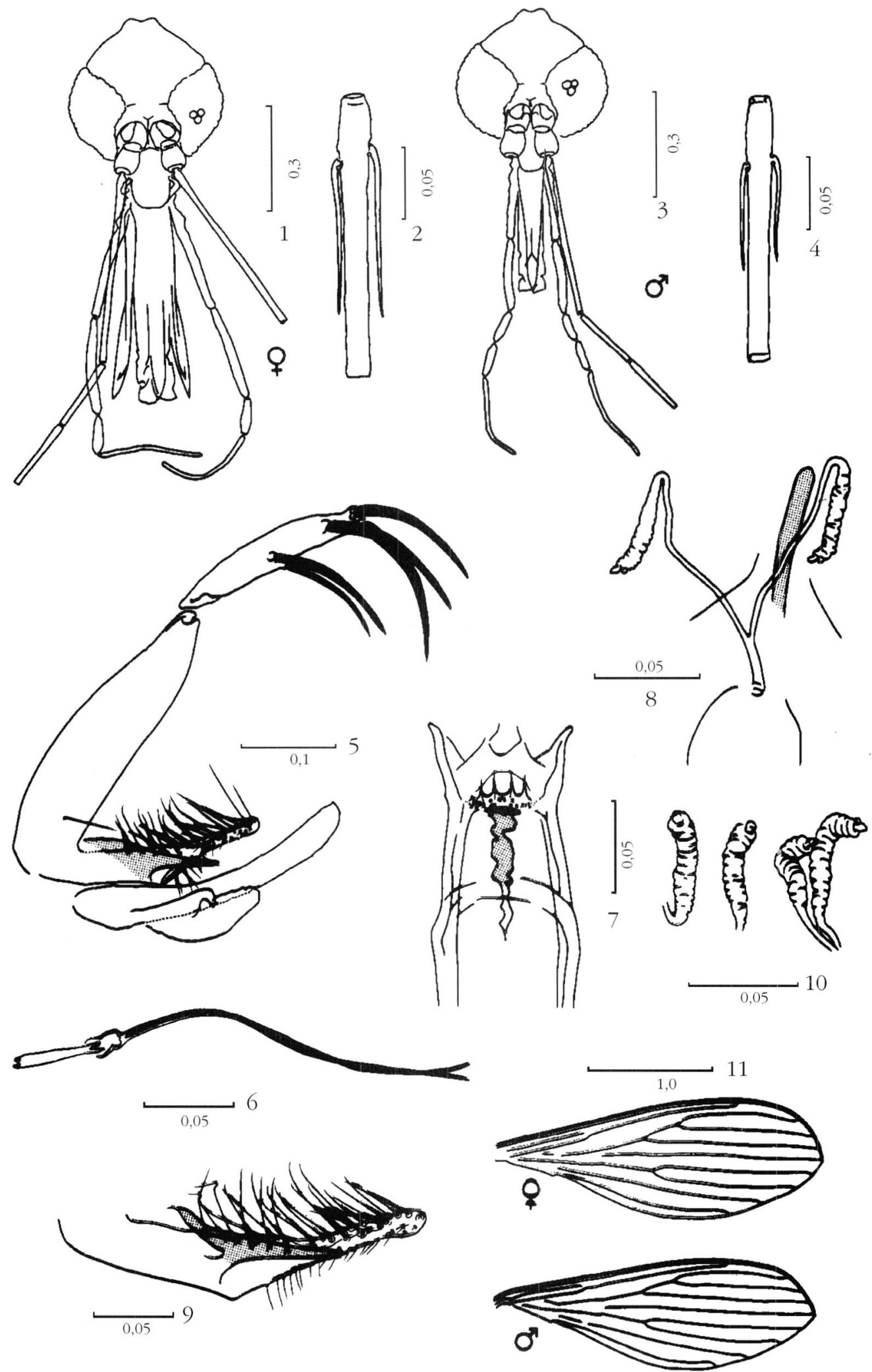

Planche I. - Lutzomyia guderiani n. sp. - Holotype mâle : - 3. tête; - 4. flagellomère antennaire A IV; - 5. genitalia; - 6. pompe et filaments génitaux; - 9. paramère; 11. aile. - Allotype femelle : - 1. tête; - 2. flagellomère antennaire A IV; - 7. cibarium; - 8. genitalia; - 10. spermathèques de paratypes; - 11. aile. 
pour ses trois séries, sanguinaria, peruensis, et osornoi, par Galati et Caceres (1994). La nouvelle espèce se range dans la série sanguinaria qui se caractérise, dans les deux sexes, par : la hauteur de la tête, équivalant ou un peu supérieure à sa largeur, un clypeus court, et des yeux de grande taille. Les mâles présentent une touffe allant jusqu'à 6 soies et des lobes latéraux plus courts que le coxite, les femelles sont plus difficiles à différencier (Galati et Caceres, 1992). Cette série se composait de 8 espèces : L. hartmanni (Fairchild et Hertig, 1957), L. sanguinaria (Fairchild et Hertig, 1957), L. botella (Fairchild et Hertig, 1961), L. scorzai (Ortiz, 1965), L. cirrita (Young et Porter, 1974), L. sp. de Pichinde, Colombie, de Young, (1979), L. tortura (Young et Rogers, 1984), et L. kirigetiensis (Galati et Caceres, 1992).

Les mâles de L. guderiani se distinguent de tous ceux de la série par une distance interoculaire plus étroite, égale au diamètre de 3,5 facettes, et un delta alaire plus grand, mesurant la moitié de alpha.

En outre, on les différencie : de $L$. hartmanni et $L$. sanguinaria par l'absence de pavillon de la pompe génitale; de L. scorzai par un labre plus court (AIII/E $=1,17$ à 1,37 contre 1,62); de L. sp. de Pichinde, par la taille du cinquième segment palpaire $\mathrm{P} 5$ inférieure à 0,30 et des filaments génitaux plus courts $(0,40-0,45$ contre 0,50$)$; de L. tortura par la taille supérieure de P5 dépassant 0,23; de L. kirigetiensis par la plus grande taille du lobe latéral $(>0,27)$ et l'absence ou l'unicité des soies du coxite; enfin, de L. cirrita par le niveau d'insertion plus apical des épines subterminale et inférieures du style, et surtout par l'absence ou la rareté de soies persistantes à la base du coxite.

Chez les femelles, le rapport alpha/delta, oscillant de 1,8 à 2 est un critère discriminant vis à vis de toutes les espèces, sauf de L. larensis (série osornoi) qui présente aussi un delta $=1 / 2$ alpha. Mais, chez cette dernière espèce, la taille du segment antennaire AIII est beaucoup plus grande $(0,66$ contre 0,44 chez $L$. guderiani).

Le sous-genre Helcocyrtomyia étant très homogène et les femelles posant toujours des problèmes de détermination en raison de la petite taille de leurs spermathèques qui passent inaperçues dans les montages non colorés, on doit systématiquement porter son attention, dans les récoltes effectuées dans une station, aux mâles ainsi qu'à la faune associée pour assurer son diagnostic; de plus, une autre complication vient de ce que la taille des spécimens varie fortement avec l'altitude (obs. pers.; Young, 1979).

L'association des sexes a été établie par : 1/ la concordance des caractères extra-génitaux et des caractéristiques morphométriques, 2/ la présence d'une seule autre espèce d'Helcocyrtomyia, voisine de $L$. castanea, dans les stations de récolte, et 3/ la présence régulière des deux sexes dans tous les piègeages effectués dans les Yungas.

L. guderiani est une espèce forestière, anthropophile, que l'on rencontre aussi sous caféière dans les Yungas, de $1400 \mathrm{~m}$ à $2000 \mathrm{~m}$ d'altitude.

Cette espèce est dédiée au docteur R.H. Guderian, éminent parasitologiste, qui nous a accueilli dans son laboratoire de Quito.

\section{LUTZOMYIA RISPAILI N. SP. (Planche II)}

DesCRIPTION DE L'HOLOTYPE MALE (paratypes, $n=10$ )

Grand phlébotome, mesurant $3,3 \mathrm{~mm}$ du bout du labre à l'extrémité du coxite, de tonalité générale brun clair.

Tête :

Longueur 0,44 $(0,409-0,47)$ incluant le clypeus; largeur (max.) $0,378(0,345-0,38)$. Rapport $\mathrm{L} / 1=1,17$. Distance interoculaire $0,1(0,08-0,11)$ courte, égale au diamètre de 5 facettes. Labre-épipharynx, longueur $0,291(0,244-0,315)$ à partir du bord du clypeus. Flagellomères antennaires : AIII 0,411 $(0,335-0,45)$, A $\mathrm{IV}+\mathrm{A} \mathrm{V}=0,171+0,167$. Rapport A III $/ \mathrm{E}=1,4(1,36-$ $1,5)$. Ascoïdes simples et courts dépassant légèrement la moitié du segment A IV (fig. 2). Palpes : longueur totale $0,725(0,637-0,827)$, longueurs respectives des palpomères : 1- 0,038 $(0,029-0,041), 2$ - $0,159(0,120$ 0,164), 3- 0,179 $(0,141-0,2), 4-0,082(0,077-0,104), 5-$ $0,267(0,233-0,35)$. Formule palpaire : 1.4.2.3.5. Cibarium présentant de nombreuses ponctuations hyalines et parfois des denticules latéraux; arc chitineux et aire pigmentée indiscernables. Pharynx postérieur à denticules crénelés, longueur 0,184 largeur (max.) 0,038 .

Thorax :

Longueur 0,61; mesonotum pigmenté, pleures claires. Mesanepisternum présentant 11 (6-17) soies hautes, et 4-5 (3-7) soies basses. Aile, longueur 2,16 (1,97$2,42)$, largeur $(\max ) 0,7(0,59-0,74)$. Rapport $\mathrm{L} / 1=$ 3,08. Indices alaires : alpha $0,73(0,64-0,8)$, beta 0,24 $(0,16-0,26)$, gamma $0,36(0,33-0,45)$, delta $0,28(0,23$ 0,31). Alpha/beta $=3,04$, alpha/delta $=2,6$. Pattes sans épines, longueurs respectives du fémur, du tibia, et du basitarse : 0,75-1,19-0,85 pour les pattes antérieures, 0,68-1,31-0,93 pour les pattes médianes, et 0,77-1,47-1,06 pour les pattes postérieures.

Abdomen :

Longueur 1,956 incluant le coxite. Coxite : longueur 0,306 (0,267-0,35), largeur (max.) 0,074 (0,065-0,075), présentant à sa base une touffe de 18 à 21 soies plus 


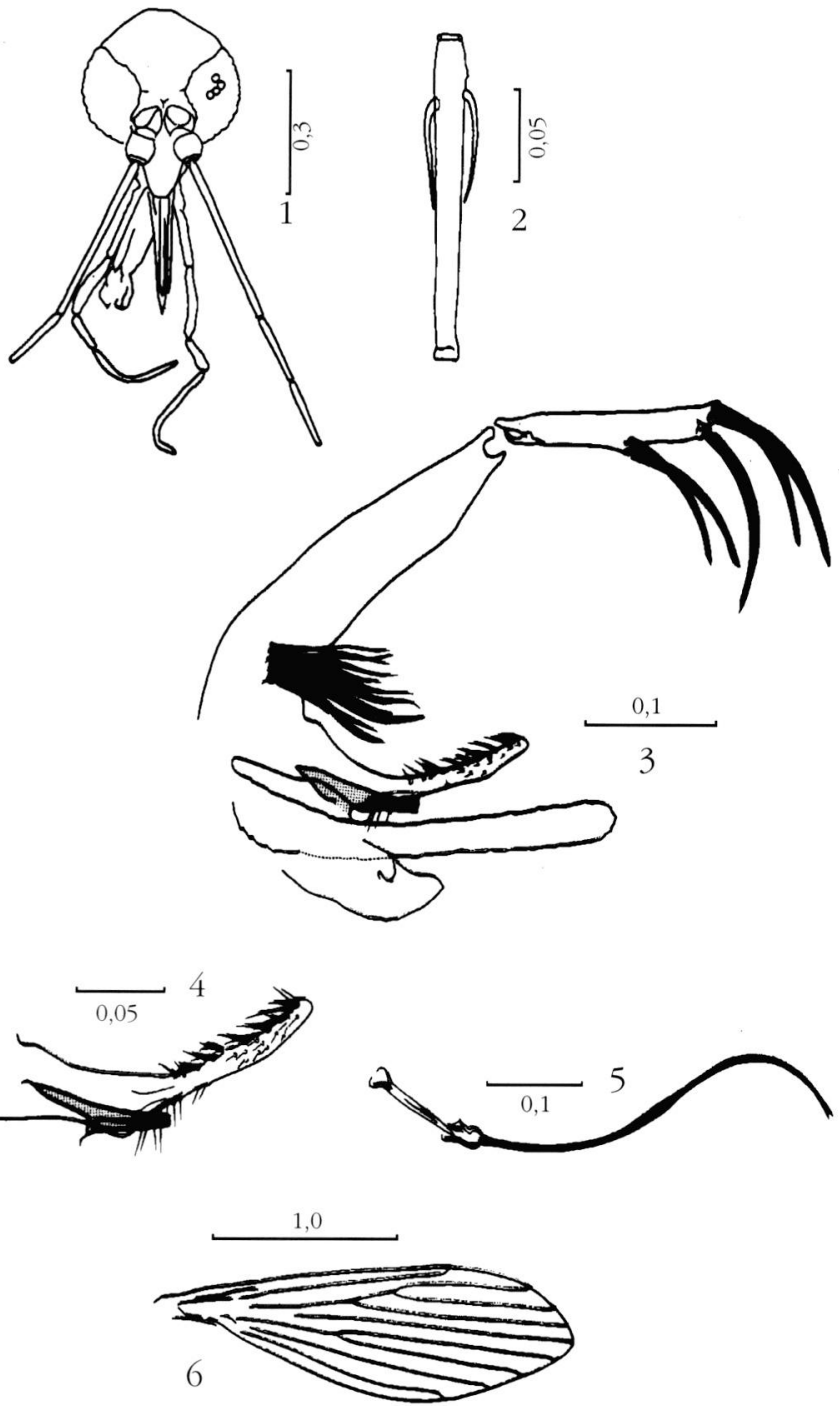

Planche II. - Lutzomyia rispaili n. sp. - Holotype mâle. - 1 . tête; -2 . flagellomère antennaire A IV; -3 . genitalia; -4 . paramère; -5 . filaments génitaux; -6 . aile.

grandes, plus foliacées, et plus courbées au centre; les extrémités des soies sont toujours aigues. Style, longueur 0,177 (0,160-0,188), largeur (max.) 0,028 (0,021-0,030), portant 5 épines : 2 terminales, 1 subterminale au tiers de la moitié apicale, et 2 inférieures, au même niveau, insérées au milieu du segment ou légèrement au-delà (vers l'apex). Paramère, longueur 0,222 (0,21-0,265), courbé en son milieu, et présentant un épaississement au niveau du 1/3 apical; bords de la moitié apicale présentant des soies spiniformes courtes, courbées antérieurement, et quelques soies fines et raides plus longues; partie apicale portant latéralement 6 à 10 soies extrêmement courtes; sur la moitié ventrale du bras, en deçà du coude, une dizaine de soies raides (fig. 4). Lobe latéral inerme : longueur 0,276 (0,261-0,29; $\mathrm{m}=0,27)$ plus court que le coxite. Lamelle sous-médiane atteignant la moitié du lobe latéral. Edéage conique. Pompe génitale d'une longueur de 0,118 (0,104-0,130). Filaments géni- taux d'une longueur de 0,457 $(0,39-0,47$ moyenne $=$ $0,42)$ soit 3,8 fois $(3,2-3,9)$ la longueur de la pompe.

\section{LOCALITÉ-TYPE ET MATÉRIEL-TYPE}

Holotype mâle (lame $n^{\circ}$ FLP-9414) et 5 Paratypes mâles (lames n FLP-9415 à 9419) récoltés au piège lumineux CDC, en sous-bois de forêt primaire, au col de Marimonos (altitude 900-1000 m) route Palos Blancos-Inicua, Alto Beni, Département de La Paz, Bolivie. Cette espèce n'a pas été capturée dans les Yungas.

\section{DISCUSSION}

Lutzomyia rispaili appartient, comme la précédente espèce, au sous-genre Helcocyrtomyia Barretto. Bien que les lobes latéraux soient de taille inférieure aux coxites, cette espèce se rapproche des espèces de la série peruensis : L. noguchii (Shannon, 1929), L. pe- 
ruensis (Shannon, 1929), L. pescei (Hertig, 1943), L. ayacuchensis (Caceres et Galati, 1988), L. blancasi (Galati et Caceres, 1990), L. tejadai (Galati et Caceres, 1990), L. pallidithorax (Galati et Caceres, 1994), par la morphologie du paramère et la présence d'une importante touffe de soies, totalement ou en partie foliacées, sur le coxite.

$L$. rispaili se sépare aisément de L. noguchii, L. blancasi, L. ayacuchensis et L. peruensis par l'implantation des deux épines inférieures du style sur la partie distale alors que chez les autres elles sont sur la partie basale, par l'insertion non pédonculée de la touffe du coxite, et par la pilosité et la morphologie du paramère. De plus, chez L. rispaili, la taille des filaments génitaux, des lobes latéraux, et du cinquième segment palpaire, sont : 0,42, 0,27, 0,28, inférieures aux valeurs correspondantes des autres espèces : 0,52 , 0,35, 0,36 (manquent les données pour L. noguchii).

La morphologie et la pilosité du paramère de L. rispaili sont proches de celles de L. tejadai. Néanmoins, il peut en être séparé par la taille plus courte du style $(0,17$ contre 0,24$)$, la forme du paramère, et la structure des soies de la touffe du coxite.

L. rispaili se distingue de $L$. pescei par la taille du labre (moyenne : 0,28), plus courte que la hauteur de la tête.

Enfin, L. rispaili se différencie de L. pallidithorax par la pigmentation plus foncée de l'insecte, les soies plus foliacées de la touffe du coxite, et les soies spiniformes, courtes et peu nombreuses du paramère.

L. rispaili est une espèce de sous-bois de forêt primaire.

Nous dédions cette espèce au docteur P. Rispail qui a contribué à éclaircir la phylogénie du genre Pblebotomus (Rispail, 1990).

\section{DÉPÔT DES TYPES}

Les spécimens des deux espèces sont déposés au Département Santé de l'ORSTOM (service Typologie et Chorologie des Vecteurs), 911 avenue Agropolis, BP 5045, 34032 Montpellier cedex. Des exemplaires seront déposés à l'Institut National des Laboratoires de Santé (INLASA; 3 mâles et 3 femelles) ainsi qu'à l'Institut Bolivien de Biologie d'Altitude (IBBA; 3 mâles et 3 femelles), à La Paz.

\section{RÉFÉRENCES}

Arana M., Evans D.A., Zolessi A., Llanos-Cuentas A. \& Arevalo J. Biochemical characterization of Leishmania (Viannia) braziliensis and Leishmania (Viannia) peruviana by isoenzyme electrophoresis. Transactions of the Royal Society of Tropical Medicine and Hygiene, 1990, 84, 526-529.
Caceres A. \& Galati E.A.B. Descriçao de Lutzomyia ayacuchensis, sp. n. (Diptera, Psychodidae, Phlebotominae) dos Andes peruanos. Revista brasiliensa Entomologica, 1988, 32, 55-61.

Blancas F., Caceres L.A.G. \& Galati E.A.B. Descriçao de Lutzomyia caballeroi, sp. n. (Diptera, Psychodidae, Phlebotominae) dos Andes peruanos. Revista brasiliensa Entomologica, 1989, 33, 455-463.

Galati E.A.B. \& Caceres A.G.L. Descriçao de Lutzomyia blancasi, sp. n. e Lutzomyia tejadai, sp. n. (Diptera, Psychodidae, Phlebotominae) dos Andes peruanos. Revista brasiliensa Entomologica, 1990, 34, 505-521.

Galati E.A.B. \& CACERES A.G. Descriçao de Lutzomyia kirigetiensis, sp. n. (Diptera, Psychodidae, Phlebotominae) do Peru e consideraçoes sobre a composiçao do subgenero Helcocyrtomyia Barretto. Revista brasiliensa Entomologica, 1992, 36, 449-456.

Galati E.A.B. \& Caceres A.G. Descriçao de Lutzomyia pallidithorax, sp. n. e de Lutzomyia castanea, sp. n. do Peru e analise cladistica das series do subgenero Helcocyrtomyia Barretto (Diptera, Psychodidae, Phlebotominae). Revista brasiliensa Entomologica, 1994, $38,471-488$.

Rispail P. Approche phénétique et cladistique du genre Phlebotomus Rondani \& Berté, 1840 (Diptera : Psychodidae). 1990, Thèse, Académie de Montpellier.

Accepté le 19 décembre 1994 